INRA Prod. Anim.,

2014, 27 (2), 89-100

\title{
Agro-écologie et écologie industrielle : deux alternatives complémentaires pour les systèmes d'élevage de demain
}

\author{
M. THOMAS ${ }^{l}$, L. FORTUN-LAMOTHE ${ }^{2,3,4}$, M. JOUVEN ${ }^{5,6}$, M. TICHIT $T^{7,8}$, \\ E. GONZÁLEZ-GARCÍA ${ }^{6,5}$, J.-Y. DOURMAD ${ }^{9,10}$, B. DUMONT ${ }^{1,12}$ \\ ${ }^{1}$ Université de Lorraine - INRA, USC0340 Animal Fonctionnalités des Produits Animaux, \\ F-54505 Vandouvre-lès-Nancy, France \\ 2 INRA, UM1388 GenPhySE, F-31326 Castanet-Tolosan, France \\ ${ }^{3}$ Université de Toulouse, INPT ENSAT, UM1388 GenPhySE, F-31326 Castanet-Tolosan, France \\ ${ }^{4}$ Université de Toulouse, INPT ENVT, UM1388 GenPhySE, F-31076 Toulouse, France \\ ${ }_{5}^{5}$ Montpellier SupAgro, UMR868 Selmet, F-34060 Montpellier, France \\ 6 INRA, UMR868 Selmet, F-34060 Montpellier, France \\ 7 INRA, UMR1048 Sadapt, 16 rue Claude-Bernard, F-75231 Paris, France \\ 8 AgroParis Tech, UMR1048 Sadapt, 16 rue Claude-Bernard, F-75231 Paris, France \\ ${ }^{9}$ INRA, UMR1348 Pegase, F-35590 Saint-Gilles, France \\ 10 Agrocampus Ouest, UMR1348 Pegase, F-35000 Rennes, France \\ 11 INRA, UMR1213 Herbivores, Theix, F-63122 Saint-Genès-Champanelle, France \\ 12 Clermont Université, VetAgro Sup, UMR1213 Herbivores, BP 10448, \\ F-63000 Clermont-Ferrand, France \\ Courriel:marielle.thomas@univ-lorraine.fr
}

Les productions animales sont à la croisée de nouveaux enjeux sociaux, économiques et environnementaux. Il s'agit de proposer de nouvelles manières de produire pour satisfaire la demande croissante en produits d'origine animale, tout en préservant l'environnement et les ressources naturelles, en augmentant l'autonomie des exploitations ou des territoires, et en valorisant les savoir-faire locaux. L'agro-écologie et l'écologie industrielle sont ici envisagées comme deux voies complémentaires pour répondre à ces enjeux ${ }^{1}$.

L'objectif de « produire mieux avec moins d'intrants » s'impose comme un nouvel horizon de l'agriculture à l'aube du troisième millénaire. La demande croissante pour les produits animaux est liée à l'augmentation de la population mondiale et à la demande accrue de protéines d'origine animale dans les pays émergents. Cet objectif doit s'accompagner d'une réduction des gaspillages, qui représentent jusqu'à $30 \%$ de l'ensemble des aliments produits chaque année dans le monde (FAO 2011). Le secteur de l'élevage doit aussi répondre aux défis environnementaux liés aux pollutions, à l'érosion de la biodiversité et plus généralement aux changements d'usage des terres, fruits de multiples facteurs d'origines anthropiques et naturels (Lecerf 2008). Par ailleurs, la demande sociétale est forte pour des aliments sains provenant de systèmes de production qui respectent le bien-être animal. Aussi des démarches telles l'intensification écolo- gique, l'agriculture à haute valeur environnementale, l'agriculture biologique ou encore l'éco-agriculture pour ne citer qu'elles, s'affichent comme des alternatives à l'agriculture conventionnelle. Ce « reverdissement » de l'agriculture est une traduction des préoccupations et de l'insatisfaction des consommateurs et de certains producteurs, vis-à-vis des productions industrielles.

A l'heure actuelle, il n'existe pas de consensus scientifique sur les moyens à mettre en œuvre pour atteindre ces objectifs, en prenant en compte la diversité des systèmes de production (allant des systèmes herbagers ou pastoraux extensifs à des systèmes industriels intensifs) et des contextes biogéographiques sous la contrainte des changements climatiques.

Dans cet article nous défendons l'idée qu'exploiter des principes et modes d'actions de l'Agro-Ecologie (AE) et de
l'Ecologie Industrielle (EI) devrait permettre de produire mieux avec moins d'intrants. Ces deux disciplines scientifiques, nées dans les années 1980 aux Etats-Unis, mobilisent les connaissances de l'écologie pour concevoir et gérer durablement les systèmes de production (cf. encadré 1). L'AE se définit aussi comme un ensemble de pratiques dont l'objectif est d'amplifier les processus naturels pour concevoir des systèmes productifs peu artificialisés, respectueux de l'environnement et moins dépendants des intrants (Altieri 1987, Gliessman 1997, Wezel et Soldat 2009). Elle met l'accent sur les interactions entre les composantes biologiques, physiques, techniques et socio-économiques des systèmes agricoles, au travers d'un mode partagé de production de connaissances et d'innovations. L'intégrité de l'écosystème - qui garantit la production sur le long terme - est aussi importante que la production d'aliments à court terme.

1 Texte adapté d'une publication en langue anglaise de Dumont et al (2013) dans la revue Animal. 


\section{Encadré 1. Les concepts de l'écologie.}

L'écologie (Haeckel 1866) étudie les interactions entre les êtres vivants et leur milieu. On peut appréhender ces interactions à trois niveaux : celui de l'individu, de la population ou des relations entre populations.

Interactions, tolérance et adaptation. Le premier principe de l'écologie est que les êtres vivants sont en relation continuelle avec leur milieu. Les caractéristiques abiotiques du milieu (lumière, température, pluviométrie, caractéristiques physiques du sol...) conditionnent leur développement. En retour, les êtres vivants s'adaptent à leur milieu de vie (adaptations morphologique, comportementale, digestive, métabolique...) afin d'augmenter leur tolérance à ses caractéristiques. Mais ces capacités d'adaptation sont limitées. Une perturbation importante peut entrainer une réduction ou la disparition d'une population. Une espèce généraliste sera capable de tolérer une diversité de conditions et ainsi de peupler des milieux différents, à l'inverse d'une espèce spécialiste qui ne pourra survivre et se développer que dans des conditions de milieu particulières.

Régulations et relations biotiques. Les interactions biotiques directes ou indirectes soustendent le fonctionnement des écosystèmes. Entre les espèces, on distingue des relations de symbiose (obligatoires pour la survie des espèces impliquées), de mutualisme (association bénéfique entre deux espèces), de commensalisme (association dont seule une espèce tire profit sans pour autant nuire à l'autre), de parasitisme (association au bénéfice du seul parasite), ou de compétition (rivalité pour l'accès aux ressources ou aux partenaires sexuels). La relation la plus fréquemment mobilisée est la relation de prédation (manger ou être mangé), qui conduit à définir des chaînes alimentaires. Au sein d'une même espèce existent des phénomènes de coopération ou de compétition, avec parfois un partage du territoire, et une organisation en société hiérarchisée. L'ensemble de ces interactions permet l'accomplissement des besoins physiologiques des espèces, leur développement et leur survie.

Réseaux trophiques. L'ensemble des chaînes alimentaires unissant les populations d'espèces que comporte une biocénose constitue les réseaux trophiques. Ce sont ces interconnexions alimentaires entre être vivants de différentes natures (producteurs primaires, consommateurs primaires ou secondaires et détritivores) qui permettent le recyclage des éléments (carbone, azote, phosphore...) et de l'énergie. La structure des réseaux trophiques influence fortement la quantité, la diversité, la stabilité et la qualité de la biomasse et de la matière organique produites par les écosystèmes.

Dynamique des écosystèmes. Populations et écosystèmes ne sont pas des structures figées mais en évolution permanente en lien avec l'évolution des facteurs du milieu et des autres populations (prédation, habitat...). Dans les systèmes agricoles, l'évolution naturelle est contrôlée ou pilotée pour favoriser le développement des espèces d'intérêt agronomique. Le maintien d'un système artificialisé appelle ainsi un compromis entre les dynamiques naturelles et les besoins pour la production agricole.

Diversité, stabilité, productivité. La diversité entre espèces d'un écosystème est d'autant plus élevée que les conditions de vie sont variées. Avec une diversité croissante, la complexification des mécanismes de régulation accroît la stabilité de l'écosystème. La stabilité des conditions environnementales permet aux écosystèmes de tendre vers un équilibre. L'énergie stockée sous forme de biomasse dans un écosystème, donc sa productivité, peut être proportionnelle à son degré d'organisation, c'est-à-dire à sa diversité et au nombre et au degré d'interconnexion entre ses éléments constitutifs.

Au final, dans un écosystème tout est lié, interdépendant et en équilibre dynamique.

L'EI explore quant à elle les possibilités de bouclage des cycles de matière et d'énergie dans une démarche transsectorielle (Frosh et Gallopoulos 1989, Frosh 1992) dans des systèmes se composant d'unités de production très contrôlées et ayant un plus faible lien au sol. Les systèmes agricoles peuvent être intégrés au sein de clusters industriels où la mutualisation des moyens et des services concourt à réduire la consommation de ressources et les émissions polluantes par une optimisation de la valorisation des déchets.

Bien que la littérature scientifique dans le domaine de l'AE soit en forte augmentation, les productions animales en ont jusqu'ici été largement écartées. Gliessman (2006) relève pourtant les nombreux rôles bénéfiques potentiellement joués par les animaux au sein des agroécosystèmes : ils produisent des aliments riches en protéines à partir de ressources non directement valorisables par l'homme (les prairies et parcours, les résidus de culture et autres sous-produits), ils contribuent indirectement à la fourniture d'autres services écosystémiques (séquestration de carbone, préservation de la biodiversité...), ils recyclent des matières potentiellement polluantes (effluents d'élevage) et ont de nombreuses fonctions culturelles et sociales (transmission de savoir-faire locaux, instrument d'épargne...). L'AE considère la biodiversité à la fois comme une ressource à préserver et comme un levier pour la production (Veen et al 2009). L'EI est basée sur la valorisation des déchets, limitant par là même les intrants et l'empreinte environnementale des Systèmes
d'Elevage (SE). Elle est encore peu sollicitée par les productions animales, à l'exception de la gestion à grande échelle des déjections dans les systèmes intensifs (Holm Nielsen 2010). Elle pourrait cependant contribuer de façon notable à la production alimentaire, avec des systèmes économes en espace, en eau et en énergie. Nous faisons l'hypothèse que l'association d'élevages relevant de l'AE (notamment pour valoriser les terres de parcours, non cultivables) et de l'EI (en particulier dans le cadre de l'agriculture périurbaine) serait un moyen de répondre aux défis de l'élevage de demain.

Cet article propose une réflexion sur l'appropriation des concepts de l'écologie et leur application pour les productions animales. La première partie décrit cinq principes agro-écologiques mobilisables pour la caractérisation de systèmes d'élevage plus durables (Dumont et al 2013), et énoncés sous l'angle d'objectifs à atteindre. Dans une deuxième partie, nous examinons quatre exemples emblématiques de production animale couvrant un large gradient d'intensification; nous montrons comment les principes précités sont combinés pour produire des services environnementaux et/ou des bénéfices économiques. Dans une troisième partie, nous abordons les implications, les opportunités et les limites des approches d'AE et d'EI pour la conception et la mise en œuvre de SE innovants.

\section{1 / Les cinq principes agro- écologiques à promouvoir pour des systèmes d'élevage innovants}

Les principes énoncés par Altieri (2002) pour une conduite agro-écologique des agro-écosystèmes (cf. encadré 2 ) ont été le point de départ de notre réflexion pour les productions animales. Tels que formulés initialement, ces principes renvoient au domaine de la protection intégrée des cultures. Nous les avons reformulés au travers de cinq grands objectifs appliqués aux productions animales : i) gérer de manière intégrée la santé animale, ii) diminuer les intrants nécessaires à la production, iii) réduire les pollutions, iv) renforcer la résilience des SE, et $v$ ) préserver la biodiversité au sein des agroécosystèmes. Pour chacun de ces principes, nous précisons s'ils s'appliquent uniquement à l'AE ou aussi à l'EI et les champs disciplinaires mobilisés.

\section{1 / Développer des pratiques de gestion intégrée pour améliorer la santé animale}

La gestion intégrée de la santé animale est un objectif visé à la fois par l'AE et 
Encadré 2. Les cinq principes proposés par Altieri (2002).

Altieri (2002) a proposé cinq principes pour le développement d'une agriculture durable, sur lesquels s'est fondée notre réflexion. Selon lui, une conduite écologique des agroécosystèmes implique de :

Renforcer leur « système immunitaire » en promouvant des actes techniques qui renforcent le contrôle naturel des bio-agresseurs,

Diminuer les impacts environnementaux en réduisant et si possible en éliminant les intrants chimiques,

Optimiser le fonctionnement métabolique des agro-écosystèmes en favorisant la décomposition de la matière organique et le recyclage des nutriments,

Jouer de manière équilibrée sur les systèmes de régulation : cycles des nutriments, cycle de l'eau, flux d'énergie, régulation des populations,

Améliorer la conservation et la régénération des sols, de la ressource en eau et de la biodiversité.

l'EI. Il s'agit de développer une vision systémique des élevages au sein desquels les animaux et leurs pathogènes interagissent continuellement. L'étude des régulations et des relations biotiques, peut ainsi nous amener à réviser les pratiques d'élevage de façon à réduire l'exposition aux agents pathogènes et la sensibilité des animaux. On cherche aussi à favoriser les comportements d'automédication et les traitements " naturels ».

L'exposition aux agents pathogènes est liée en partie à la concentration des animaux. En AE, où le lien au sol est marqué, il est possible de perturber les cycles hôte-pathogène en jouant sur la conduite du pâturage (répartition des animaux dans l'espace et dans le temps) et grâce au pâturage mixte. En EI où les animaux sont élevés dans des systèmes en confinement, il est possible de diminuer la charge pathogène de l'environnement en raisonnant 1'occupation des bâtiments (densité d'animaux, succession des bandes...) et les échanges avec l'extérieur (biosécurité).

La sensibilité des animaux aux agents pathogènes dépend avant tout du choix du génotype à élever dans un milieu donné, avec un mode d'élevage donné. Les caractéristiques d'adaptation des individus à l'environnement d'élevage peuvent être morphologiques ou anatomiques (petite taille, faible gras souscutané, absence de plumes qui rendent les animaux moins sensibles à la chaleur), physiologiques (aptitude à valoriser des fourrages grossiers, à mobiliser les réserves corporelles face à des restrictions alimentaires...) ou comportementales (aptitude à la marche...). Le choix raisonné d'une espèce et d'une race peut permettre de limiter les intrants médicamenteux, dont l'utilisation massive se traduit par une augmentation de la fréquence d'apparition de résistances. A titre d'exemple, les travaux les plus significatifs en zone tropicale portent sur la trypanotolérance, la résistance aux maladies transmises par les tiques, et les strongyloses gastro-intestinales (Mandonnet et al 2011). Le microbiote digestif est aussi une cible privilégiée pour une gestion intégrée de la santé animale. Par exemple, l'adoption des lapereaux à l'âge d'un jour favorise la mise en place d'une flore microbienne adaptée qui réduit leur sensibilité aux maladies. Au-delà des caractéristiques propres à l'animal, la conduite d'élevage joue également un rôle. On sait notamment que le stress augmente la sensibilité des animaux; ainsi, par exemple, limiter les ré-allotements réduit la fréquence des traitements vétérinaires et permet une meilleure croissance des animaux (Mounier et al 2006).

En termes d'automédication, les avancées dans le domaine de la nutrition sont à explorer. L'exemple qui consiste à faire consommer aux animaux des plantes riches en composés bioactifs, tels que les tanins condensés, montre la possibilité de réduire de manière naturelle l'infestation des petits ruminants par les strongles digestifs (Hoste et al 2006). Autre exemple en aquaculture, l'utilisation de probiotiques naturels administrés dans les aliments ou directement dans l'eau des bassins peut améliorer la santé et la croissance des alevins, et augmenter leur résistance aux maladies (Merrifield et al 2010).

\section{2 / Potentialiser l'utilisation des ressources naturelles et des copro- duits pour diminuer les intrants nécessaires à la production}

Le deuxième principe agro-écologique vise à diminuer les intrants nécessaires à la production. Pour ce faire, deux options sont possibles : i) augmenter l'efficacité alimentaire des animaux, ii) augmenter la part des ressources non directement valorisables par l'homme dans l'alimentation animale.

Le premier levier, pouvant s'inscrire aussi bien en AE qu'en EI, consiste à améliorer l'efficacité alimentaire des animaux au travers de la sélection génétique. Raisonner le rythme de distribution des aliments permet également d'augmenter l'efficacité alimentaire, comme cela a été montré chez des poules pondeuses (Faruk et al 2010). Des avancées dans le domaine de la nutrition animale et de la physiologie peuvent être également mobilisées de façon à recourir à des ingrédients favorisant la digestion des nutriments. Pour répondre à une démarche agro-écologique, il faut dans tous les cas chercher à potentialiser les processus naturels ou à recourir à des ingrédients d'origine naturelle. En EI, l'utilisation d'additifs ou de compléments permet d'améliorer la digestibilité ou la rétention des nutriments, comme par exemple l'utilisation de phytases d'origine microbienne chez le porc (Dourmad et al 2009).

Le second levier, mobilisable à la fois en $\mathrm{AE}$ et en $\mathrm{EI}$, consiste à valoriser les prairies (permanentes ou non), les parcours, les résidus de cultures mais aussi les coproduits de l'industrie agro-alimentaire, ce qui implique de préciser leur valeur alimentaire et leurs conditions d'utilisation pour différents types d'animaux. Ainsi, il s'agit de limiter autant que possible l'utilisation de ressources limitantes comme les surfaces agricoles ou l'eau. Dans les SE basés sur des ressources cultivées, il s'agit a minima d'encourager le développement de ressources fourragères mieux adaptées à des épisodes de sécheresse (sorgho, luzerne ou graminées à dormance estivale). Augmenter la part des légumineuses dans les prairies permet de réduire la fertilisation minérale, tout en assurant des hauts niveaux de production pour les vaches laitières (Soder et al 2006).

Plus globalement, il s'agit de préserver de manière naturelle les services de support à la production et les services de régulation (stockage de carbone, régulation du cycle de l'eau...) des écosystèmes mobilisés. Ainsi la diversité fonctionnelle des prairies peut entraîner une plus grande stabilité de la production fourragère et renforce la résistance des prairies aux adventices (Lüscher et al 2008, Frankow-Lindberg et al 2009). Dans les rotations, les prairies préservent la matière organique du sol et augmentent le rendement des cultures qui les suivent. Pour les milieux aquatiques, il est également possible de stimuler la productivité des étangs grâce à des substrats immergés et en contrôlant de manière naturelle le ratio $\mathrm{C} / \mathrm{N}$ de l'eau (Bosma et Verdegem 2011). Dans tous les cas, l'exploration 
de ces multiples voies nécessite une expertise en analyse des agro-écosystèmes.

\section{3 / Optimiser le fonctionnement des systèmes d'élevage pour réduire les pollutions}

Pour réduire les pollutions des SE, il est possible d'agir à l'échelle de l'individu, essentiellement en limitant les rejets, et/ou à l'échelle de l'exploitation, en limitant les pertes ou bien en favorisant les recyclages et épurations naturelles.

Dans le premier cas, il s'agit de mobiliser des leviers alimentaires (en termes qualitatif et quantitatif). Cette voie est particulièrement envisageable dans une démarche d'EI. Ainsi, à titre d'exemple, on montre que des additifs alimentaires d'origine naturelle réduisent significativement les émissions de méthane des ruminants (Martin et al 2010), et que chez le porc l'alimentation séquencée limite l'excrétion d'azote et de phosphore (Dourmad et al 2009).

Dans le deuxième cas, il est possible d'agir tant sur la conception des bâtiments d'élevage et de stockage que sur des pratiques d'entretien et de fertilisation des surfaces. Par exemple, le maintien des prairies dans des systèmes relevant de l'AE augmente la capacité du milieu à stocker le carbone et limite le lessivage de l'azote et le ruissellement du phosphore. En aquaculture, le lagunage, la phyto-épuration et la sédimentation permettent une gestion naturelle des polluants, des voies mobilisables aussi bien en AE qu'en EI. Dans les exploitations de polyculture-élevage, l'enjeu est de favoriser le recyclage des déjections en tirant parti de la complémentarité entre différents ateliers, voire en développant de nouvelles activités comme par exemple la production d'énergie par méthanisation. Il s'agit de travailler au bouclage des cycles pour concevoir des SE peu polluants et plus autonomes. Par exemple, les systèmes d'aquaculture intégrés associant différentes espèces sont basés sur la récupération des nutriments non utilisés par les poissons, qui permet une production simultanée de crustacés ou de mollusques bivalves ; les algues cultivées au sein du système contribuent au maintien de la qualité de l'eau (Neori et al 2004). Parfois les déjections d'une espèce deviennent même un des constituants de la ration d'une autre espèce. Ainsi, en Afrique de l'Ouest, les fientes de poulet permettent de complémenter efficacement une ration à base de fourrages grossiers destinée à des chèvres naines (Alikwe et al 2011). L'enjeu est alors de limiter les risques de contamination microbienne des produits. En Asie du Sud-Est, ceci s'est traduit par des recommandations sanitaires de l'Organisation Mondiale de la Santé (WHO 2006) en vue de la commercialisation de poissons produits dans des systèmes aquacoles intégrés avec de la riziculture et des activités d'élevage.

\section{4 / Gérer la diversité des res- sources et la complémentarité des animaux pour renforcer la rési- lience des systèmes d'élevage}

La gestion spatio-temporelle de la diversité des ressources et de la complémentarité des animaux peut permettre de renforcer la résilience des SE à des aléas climatiques ou économiques (Tichit et al 2011). Ainsi la biodiversité domestique ou planifiée (i.e. les cultures et les animaux d'élevage) peut être pilotée tout en valorisant la biodiversité associée aux agro-écosystèmes (i.e. l'ensemble des autres organismes qu'ils abritent). Ceci implique d'adapter le cycle de production pour mettre en adéquation la saisonnalité des besoins alimentaires des animaux avec la diversité des ressources présentes sur l'exploitation, et d'utiliser leurs capacités d'adaptation (par exemple en utilisant le phénomène de croissance compensatrice). La production d'agneaux sur parcours décrite dans la partie 2 illustre ce principe. En Bretagne, les fermes laitières du RAD (Réseau Agriculture Durable) qui regroupent les vêlages pour maximiser la part du pâturage dans les stratégies d'alimentation ont eu des résultats économiques et environnementaux améliorés (Le Rohellec et al 2009).

Des leviers existent également à l'échelle du troupeau où il s'agit de tirer parti de la variabilité individuelle et de la diversité des trajectoires adaptatives des individus. Le domaine d'application est plus spécifiquement celui de l'AE, car cette variabilité prend tout son intérêt lorsque le milieu d'élevage est luimême variable. Ainsi, le pâturage mixte de deux espèces d'herbivores augmente l'utilisation de la biomasse disponible et réduit les risques d'infestation parasitaire ; la croissance des animaux est généralement meilleure. Valoriser la complémentarité des régimes et des stratégies alimentaires de différentes espèces augmente également la productivité des étangs de polyculture (Rahman et al 2006). Le concept de " généraliste-spécialiste » propose un cadre pour gérer la diversité des réponses individuelles. Les animaux généralistes produisent moins mais sont plus robustes. La constitution des lots et leur alimentation pourrait à terme se fonder sur une analyse du caractère généraliste ou spécialiste des animaux qui les composent (Puillet et al 2010). A l'échelle du système, élever simultanément plusieurs espèces réduit aussi les risques climatiques, sanitaires ainsi que ceux liés aux fluctuations du marché (Tichit et al 2004, Bonaudo et al 2014). Aussi, pour atteindre les objectifs posés par ce principe, des compétences en écologie mais également en nutrition animale sont à mobiliser.

\section{5 / Adapter les pratiques d'éle- vage de manière à préserver la biodiversité et à assurer les ser- vices écosystémiques associés}

Si les agro-écosystèmes doivent bien évidemment assurer une fonction d'approvisionnement, ils ont aussi à garantir tout un ensemble d'autres services écosystémiques, lesquels sont en grande partie fondés sur la préservation de la biodiversité. Les services de régulation (comme la pollinisation, le recyclage de la matière...) ou culturels et sociaux (comme le maintien de paysages attractifs) sont autant d'exemples pouvant être soutenus par la préservation de la biodiversité au sein des agro-écosystèmes. Le domaine d'application de ce dernier principe est clairement celui de l'AE. Il s'agit en pratique d'adopter des pratiques ou des conduites d'élevage (à l'échelle du système) qui permettent d'assurer le renouvellement des ressources biologiques (naturelles et sélectionnées) de manière à conserver un potentiel intrinsèque d'adaptation du système à des aléas (climatiques, sanitaires...) et à de nouvelles formes d'élevage.

Ainsi, adapter le mode d'utilisation des prairies et parcours, des assolements ou des parcelles en agroforesterie, permet de préserver leur biodiversité floristique et faunistique. Au pâturage, le maintien de l'hétérogénéité structurale du couvert et les pratiques favorisant la floraison des prairies (telles que les fauches tardives) sont bénéfiques pour l'entomofaune. Exclure du pâturage certaines parcelles au moment du pic de floraison profite ainsi aux papillons et aux bourdons sans pour autant nécessiter de réduire le chargement animal (Farruggia et al 2012, Scohier et al 2013). Il existe plus de degrés de liberté dans la résolution des compromis entre production et préservation de la biodiversité à l'échelle du système fourrager. Par exemple, éviter d'exploiter au printemps entre 20 et $50 \%$ de la surface d'une exploitation peut compenser les effets délétères d'un fort chargement sur les autres parcelles (Franzén et Nilsson 2008). Il est possible de maintenir la biodiversité en agencant le parcellaire et les usages de façon à créer des zones réservoirs, des complémentarités entre habitats et une connectivité entre milieux semi-naturels. Il a ainsi été montré que favoriser l'hétérogénéité d'un paysage herbager en modifiant la distribution des pratiques de fauche et de pâturage offrait un levier efficace pour concilier la production 
herbagère et la préservation des oiseaux limicoles dans les marais de l'Ouest de la France (Sabatier et al 2010, 2014).

La préservation de la biodiversité fait aussi appel à la diversité génétique des animaux domestiques élevés (ou des variétés cultivées) dans les agro-écosystèmes. Cela passe notamment par l'utilisation de races (variétés) locales dites rustiques, qui contribuent fortement à l'identité des territoires. Du fait de leur adaptation au milieu, les animaux de race rustique permettent de réduire les charges d'alimentation, de limiter les interventions sur le troupeau (par exemple aux périodes de mise bas) et les frais vétérinaires. Préserver les races locales est d'autant plus important que l'érosion du patrimoine génétique est très marquée dans les races fortement sélectionnées.

\section{2 / Analyse de quatre situa- tions emblématiques mobi- lisant ces principes}

Les interactions entre composantes du système et la dynamique des processus écologiques constituent des éléments qu'il faut analyser de manière intégrée pour comprendre les logiques et les relations entre pratiques et performances des $\mathrm{SE}$ agro-écologiques. Aussi, sans chercher à être exhaustif, nous illustrons comment plusieurs des cinq principes que nous venons de développer sont mobilisés dans quatre études de cas : deux relèvent clairement de démarches agro-écologiques (les systèmes intégrés agriculture-aquaculture et un système de production ovine). L'étude de cas relative à la production porcine est à rattacher à l'EI et celle sur l'élevage de palmipèdes se situe à l'interface des deux cadres d'analyse. Nous examinons quels principes agro-écologiques sont à chaque fois mobilisés (tableau 1) et considérons les performances du système et son empreinte environnementale à partir des indicateurs disponibles. Notons que dans ce même numéro spécial, l'article de Delaby et al aborde le cas des élevages bovins laitiers à bas niveaux d'intrants, et l'article de Labbé et al celui des productions piscicoles en eau recirculée, qui constituent également des exemples mobilisant l'AE et/ou l'EI.

\section{1 / Systèmes Intégrés Agriculture -Aquaculture (systèmes IAA)}

Les étangs piscicoles gérés de façon non intégrée affichent - selon les zones géographiques - une productivité naturelle comprise entre 200 et $800 \mathrm{~kg}$ de poissons/ha/an, des niveaux jugés non satisfaisants par les producteurs (Prein 2002). L'intégration de l'aquaculture avec d'autres activités agricoles amé- liore la productivité naturelle des étangs tout en préservant l'environnement. Particulièrement développés en Asie du Sud-Est, ces systèmes (figure 1) associent des productions de végétaux (fruits,

céréales), d'animaux terrestres (porcs, ruminants, volailles) et piscicoles dans une même unité d'exploitation. Les principes agro-écologiques mobilisés dans ces systèmes portent sur l'optimisation

Tableau 1. Mobilisation des principes agro-écologiques dans les quatre études de cas relevant d'une démarche agro-écologique, d'écologie industrielle ou mixte.

\begin{tabular}{|c|c|c|c|c|}
\hline & $\begin{array}{l}\text { Systèmes } \\
\text { IAA }^{(1)}\end{array}$ & $\begin{array}{l}\text { Ovins viande } \\
\text { sur parcours }\end{array}$ & Palmipèdes & $\begin{array}{c}\text { Production } \\
\text { porcine }\end{array}$ \\
\hline \multicolumn{5}{|l|}{ Principes mobilisés } \\
\hline $\begin{array}{l}\text { Améliorer la santé } \\
\text { animale }\end{array}$ & & & & * \\
\hline $\begin{array}{l}\text { Diminuer les intrants } \\
\text { nécessaires à la } \\
\text { production }\end{array}$ & $* \star *$ & ** & ** & $* * *$ \\
\hline Réduire les pollutions & ** & * & $* * *$ & $* * *$ \\
\hline Renforcer la résilience & $* *$ & $* * *$ & ** & * \\
\hline $\begin{array}{l}\text { Préserver la } \\
\text { biodiversité }\end{array}$ & & ** & & \\
\hline \multicolumn{5}{|l|}{ Nature de la démarche } \\
\hline Agro-écologie & $x$ & $x$ & $X$ & \\
\hline Ecologie industrielle & & & $x$ & $x$ \\
\hline
\end{tabular}

(1) IAA : Intégration agriculture - aquaculture

Le nombre d'étoiles traduit le niveau de mobilisation des principes :

${ }^{\star}$ : marginal ; ${ }^{* *}$ : important ; ${ }^{* * *}$ : très important

Figure 1. Diagramme simplifié des interactions entre composantes d'un système Intégré Agriculture-Aquaculture (Système IAA).

La couleur des flèches précise le principe agro-écologique mobilisé (rouge : diminution des intrants ; noir : réductions des pollutions ; gris : renforcement de la résilience).

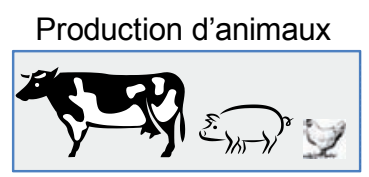

(Ruminant, cochon, volaille...)

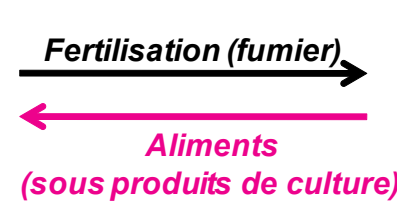

Production végétale

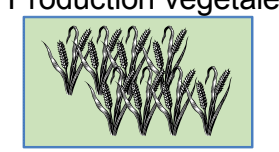

(Céréales, fruits, riz...)
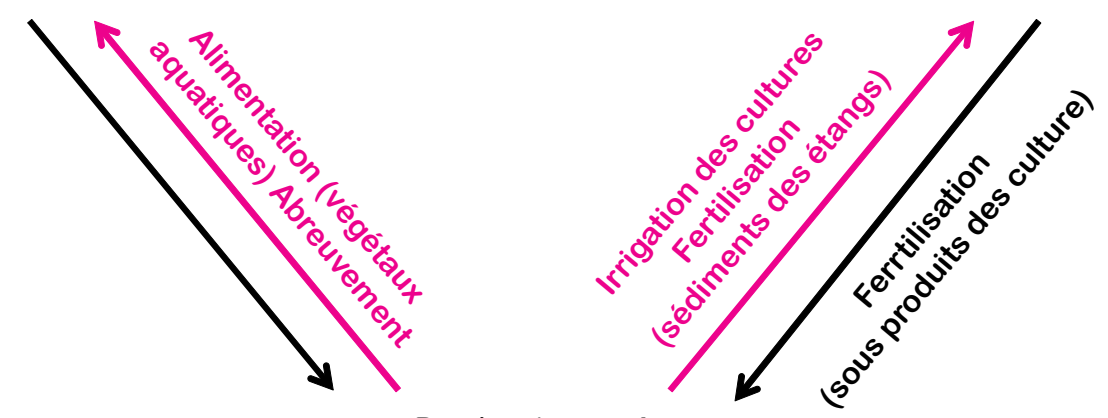

Production en étang
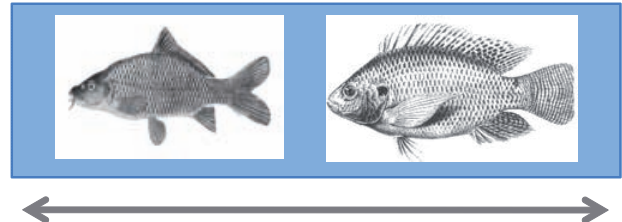

Assemblage de différentes espèces de poissons (carpe, tilapia...) 
de l'utilisation des ressources disponibles pour diminuer les intrants nécessaires à la production et sur la réduction des pollutions. Ces systèmes utilisent la diversité des ressources et la complémentarité des animaux, au profit de leur résilience (figure 1 et tableau 1).

Les systèmes IAA développés dans le delta du Mékong en sont une illustration (Nhan et al 2006, 2007 ; Phong et al 2010). Ils associent des productions de poissons, de fruits et de riz, dont les rendements varient selon la typologie des systèmes et les pratiques mobilisées. Dans une première catégorie de systèmes, les poissons sont élevés dans une série de fossés étroits et peu profonds ( 2 à $3 \mathrm{~m}$ de large et 0,5 à $0,8 \mathrm{~m}$ de profondeur) au milieu des vergers, dans lesquels peu d'intrants sont apportés. Ces fossés constituent des réservoirs d'eau servant à irriguer les vergers. On y extrait également des sédiments riches en nutriments pour fertiliser les cultures. Les rendements piscicoles sont de l'ordre de 0,5 à $2 \mathrm{t} / \mathrm{ha} / \mathrm{an}$ et la production fruitière peut dépasser 5 t/ha/an grâce aux sédiments utilisés qui apportent une fertilisation supérieure ou égale à $100 \mathrm{~kg}$ $\mathrm{N} /$ ha/an. D'autres systèmes privilégient la production de riz couplée à celle de fruits, mais avec des rendements moindres que précédemment $(<2 \mathrm{t}$ de fruits/ ha/an et une fertilisation $\leq 50 \mathrm{~kg} \mathrm{~N} / \mathrm{ha} /$ an). Les poissons sont alors produits dans des rizières ou en étangs de polyculture (5 à $30 \mathrm{~m}$ de large et plus d'un mètre de profondeur) dans lesquels on apporte de façon modérée des intrants à partir des ressources disponibles sur le site (comme les résidus de culture). Les rendements piscicoles sont de l'ordre de 2 à $10 \mathrm{t} / \mathrm{ha} / \mathrm{an}$. Les végétaux aquatiques qui se développent dans les étangs peuvent aussi être utilisés pour nourrir le bétail.

Valoriser les déchets et diversifier la production agricole constituent ainsi des leviers essentiels pour les systèmes IAA. Ceux-ci augmentent le ratio entre les productions animales/végétales et les intrants (Kumaresan et al 2009) et apportent des bénéfices économiques substantiels. Karim et al (2011) ont calculé que l'introduction de Tilapia sp. dans des systèmes intégrés augmentait nettement la marge brute par foyer qui, en zones périurbaines au Bangladesh, passe de 50-150 à 300 \$US. Par ailleurs, des analyses de cycle de vie ont permis de comparer l'efficience des systèmes IAA du delta du Mékong (Phong et al 2011). Les besoins énergétiques sont deux fois plus faibles dans le second type de systèmes associé à la riziculture $(14,2 \mathrm{~kJ} / \mathrm{kcal}$ de produit agricole) que dans le premier associé aux productions fruitières $(27,1 \mathrm{~kJ} / \mathrm{kcal})$. Dans ces systèmes intégrés, les impacts environnemen- taux (réchauffement climatique potentiel, utilisation des sols...) sont 35 à $45 \%$ plus faibles par kg de protéines de poisson que par $\mathrm{kg}$ de protéines de porc ou de volaille.

\section{2 / Un système ovin-viande sur parcours}

L'utilisation des parcours est souvent considérée comme peu compatible avec de bonnes performances zootechniques et économiques. L'expérience du troupeau ovin allaitant du domaine INRA de la Fage (Aveyron, France) prouve qu'en organisant le cycle de production animale et le système d'alimentation en fonction de la diversité des ressources, on peut concevoir des systèmes ovins productifs et qui présentent divers intérêts environnementaux.

Le troupeau de la Fage comporte 280 brebis mères de race Romane élevées en plein air intégral sur parcours. Cette race combine des caractères de rusticité et une forte prolificité $(>2,4)$ qui permet une productivité numérique élevée avec un seul agnelage par an (Molénat et al 2005). Les 50 agnelles de renouvellement sont mises à la reproduction tardivement (19 mois), pour atteindre un poids suffisant à la mise à la reproduction $(>40 \mathrm{~kg})$ malgré une croissance ralentie et/ou discontinue. Les animaux exploitent 278 ha de parcours, 10,2 ha de prairies cultivées (associations graminées-légumineuses) fauchées et pâturées et 2,8 ha de céréales. Dix-huit hectares de parcours sont fertilisés depuis plus de 30 ans $(30 \mathrm{~kg} \mathrm{~N}$ minéral/ha/an) ; la végétation y est précoce et la production trois fois plus élevée que sur le parcours «natif» (environ $6 \mathrm{t}$ contre $2 \mathrm{t} \mathrm{MS/ha/an).}$

L'agnelage débute avec le démarrage de l'herbe sur le parcours fertilisé, où les brebis suitées de 1 à 3 agneaux trouvent une herbe de qualité. Les agneaux sont sevrés en fin de printemps, à 70 jours. Ils seront engraissés avec les repousses de cultures, du foin et du concentré (en partie autoproduit). Les agnelles pâturent les parcs les plus escarpés et embroussaillés au moment où la qualité de l'herbe est la meilleure. Durant l'été, les brebis taries finissent l'herbe laissée par les agnelles. En automne, le troupeau pâture à nouveau les parcs de printemps, puis finit les repousses sur les cultures, pour reprendre de l'état avant l'hiver, période qui cumule forts besoins (fin de gestation) et conditions environnementales défavorables. En hiver, le troupeau pâture le report sur pied sur des parcs peu ou pas utilisés le reste de l'année. Lorsque l'herbe se fait rare, une complémentation en fourrages est apportée. En fin de gestation, des céréales (autoproduites) sont progressivement introduites dans la ration. On cherche ainsi à valoriser les capacités d'adaptation des animaux et la diversité des ressources pour accroître la résilience du système (tableau 1). Ce faisant on diminue les intrants tout en préservant un milieu riche en biodiversité.

Les performances de ce système ont d'abord été évaluées par modélisation (Jouven et al 2010). Les chiffres ci-après correspondent aux résultats de cette simulation. La productivité numérique simulée est de 1,8 agneau par femelle de plus de 12 mois. Les parcours fournissent près de $70 \%$ de l'alimentation et l'exploitation est autonome à $93 \%$. Le revenu net (24,6 $\mathrm{k} €$ par an) est supérieur aux références enregistrées pour les exploitations ovins viande du réseau « herbager Massif-Central » et stable entre années. Le système entretient les parcours et consomme peu d'énergie non renouvelable (56 MJ/kg carcasse, un niveau inférieur de $25 \%$ aux systèmes herbagers « moyens » dans les références de l'Institut de l'Elevage - IDELE 2010). Les bilans économiques et environnementaux réalisés depuis 2010 confirment ces prévisions.

\section{3 / Agroforesterie et méthanisa- tion pour la production de foie gras d'oie}

Dans le système de palmipèdes que nous décrivons dans cette section, les pratiques agro-écologiques combinent l'utilisation d'animaux de souches locales, le choix des rations alimentaires adaptées, la complémentarité entre l'élevage et l'agroforesterie et la méthanisation des déjections (figure 2). Trois principes agro-écologiques sont principalement mobilisés dans cet exemple (tableau 1) à l'interface entre $\mathrm{AE}$ et $\mathrm{EI}$ : en premier lieu une réduction des pollutions, mais aussi une diminution des intrants et un renforcement de la résilience du système, en lien avec la production simultanée d'oies et de noix.

La ferme expérimentale de l'oie (Coulaures, Dordogne) élève 550 oies reproductrices, 200 mâles et 4 à 5 bandes annuelles de 1200 oies en croissance sur 15 ha de pâturages boisés. Une salle de gavage permet de gaver des bandes de 300 animaux pour la production de foie gras. Une unité de méthanisation sur la ferme produit $500000 \mathrm{~kW} / \mathrm{h} / \mathrm{an}$ d'électricité et chauffe les bâtiments d'élevage, à partir des déchets de l'élevage, et des élevages et usines aux alentours.

Les oies reproductrices reçoivent un aliment complet sous forme de granulés et sont logées en bâtiment. Elles ont accès à 1 ha de parcours herbagé non fertilisé sous couvert de noyers. Leurs déjections augmentent les taux de matière 
Figure 2. Complémentarité entre les productions animales et végétales, le recyclage et la valorisation des éléments dans une exploitation avicole.

La couleur des flèches précise le principe agro-écologique mobilisé (rouge : diminution des intrants ; noir : réductions des pollutions ; gris : renforcement de la résilience).

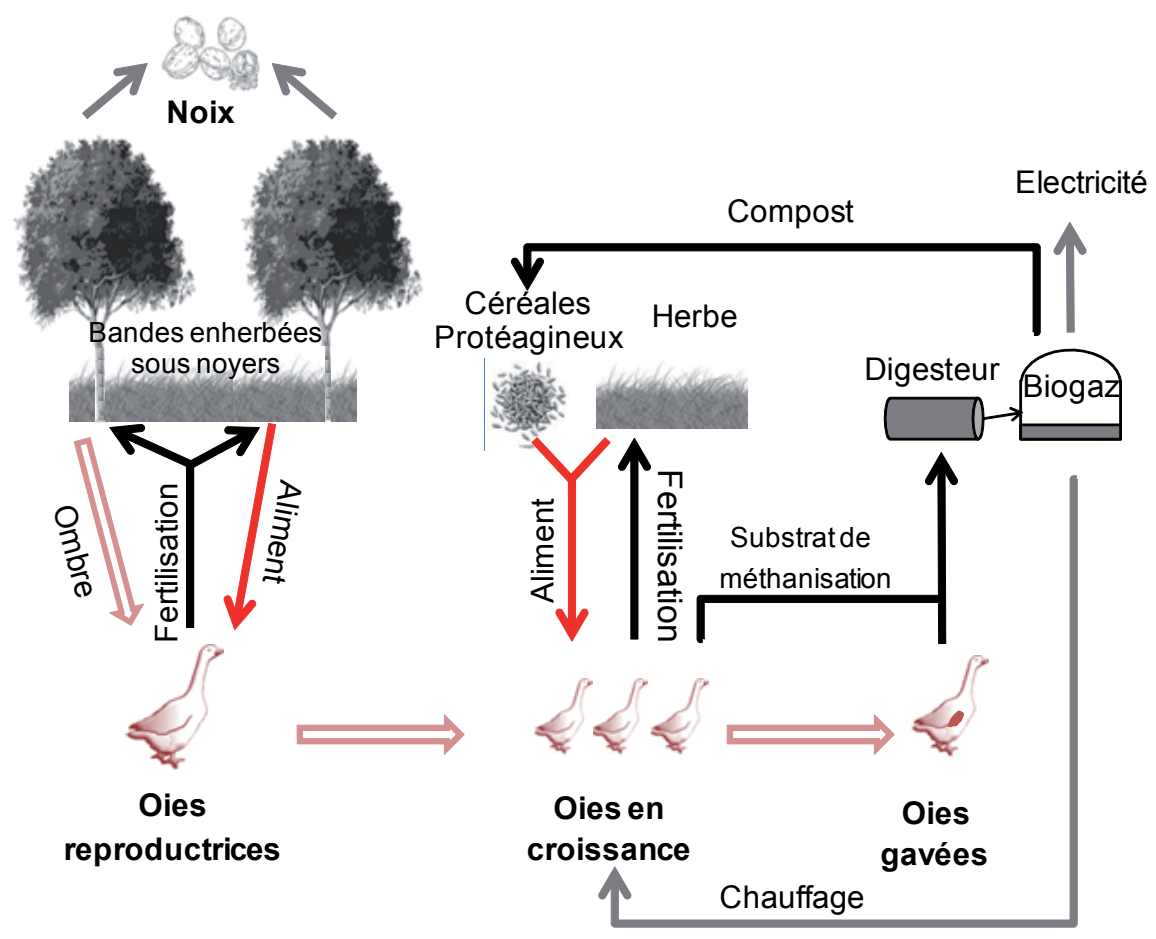

organique (66 vs $58 \mathrm{~g} \mathrm{MO} / \mathrm{kg}$ sans animaux), d'azote $(2,8$ vs $2,5 \mathrm{~g} \mathrm{~N} / \mathrm{kg})$ et de phosphore $(0,77$ vs $0,49 \mathrm{~g} \mathrm{P} / \mathrm{kg})$ du sol (Bijja et al 2010). Cette association améliore la production de noix de $26 \%$ et la croissance des arbres de 6\% (Dubois et al 2008a). Les risques de contamination microbienne des fruits (par Escherichia coli) sont écartés si les oies sont retirées du parcours au moins deux mois avant la récolte. Par temps chaud, la plupart des animaux (jusqu'à $94 \%$ à $14 \mathrm{~h} 00$ ) profitent de l'ombre apportée par les noyers (Dubois et al 2008b).

Au démarrage, les oisons sont élevés en bâtiment pour assurer leur confort thermique. A partir de 6 semaines, ils ont accès à un parcours herbagé de dactyle, pâturé en rotation. Cette gestion du pâturage (par rapport à un élevage en bâtiment ou en pâturage continu) améliore l'état des animaux (qualité du plumage et état des pattes) tout en contribuant aux apports nutritionnels, l'ingestion journalière d'herbe fraîche pouvant atteindre $1,2 \mathrm{~kg}$ par oie (Leprettre et al 2000). L'enherbement du parcours réduit également les phénomènes de lixiviation. L'essentiel des apports alimentaires provient néanmoins de céréales et de protéagineux. Ces derniers peuvent être apportés sous forme d'un mélange fermier composé de graines cultivées localement (féverole, lupin, maïs et sorgho), concassées ou bien en mélange de céréales entières complétées par un granulé riche en protéines (Arroyo et al 2012). Ces apports permettent des performances clés de la maîtrise des impacts environnementaux.

L'exemple décrit ci-après s'inscrit dans une démarche d'optimisation du fonctionnement métabolique d'une exploitation, qui s'inspire des principes de l'écologie industrielle. Il montre les relations avec d'autres éléments du territoire. Il s'agit d'un élevage naisseur-engraisseur de 180 truies situé à Fléré la Rivière (Indre), dans lequel les effluents sont méthanisés puis utilisés pour la fertilisation des 252 ha de cultures de céréales, oléagineux et pois. Les cultures sont utilisées pour alimenter les porcs, à l'exception du tournesol, du millet et du sarrasin qui sont vendus. Le digesteur est principalement approvisionné par les effluents liquides et solides et par des résidus d'inter-cultures, mais il utilise aussi les tontes des pelouses communales. La méthanisation permet de réduire les émissions de $\mathrm{CH}_{4}$ dans l'environnement et diminue l'odeur des digestats et leur charge en bactéries comme en résidus (Petersen et al 2007). Le biogaz est utilisé pour la production d'électricité (880 MWh électrique soit la consommation de 250 habitations) et de chaleur (847 thermal MWh thermique). Le colza est pressé pour la production d'huile transformée en énergie et de tourteau utilisé comme source de protéines pour l'alimentation des porcs. La chaleur produite sert au chauffage des bâtiments, réduisant ainsi son coût et les émissions de gaz à effet de serre (Rigolot et al 2010). Les aliments sont fabriqués sur place, principalement à partir des ingrédients produits sur l'exploitation qui constituent en moyenne $75 \%$ de la ration. Les performances techniques de l'élevage sont voisines de la moyenne française, supérieures en engraissement et un peu inférieures en reproduction. Les variations annuelles de la marge brute sont fortement atténuées par l'autoproduction d'une part importante des aliments, la vente d'une partie des cultures, et par la transformation et la vente directe de charcuterie. L'exploitation emploie ainsi 8,5 UTH pour l'ensemble de ses activités. Cet exemple illustre bien l'intérêt qu'il peut y avoir à mobiliser les principes agro-écologiques (tableau 1) pour favoriser les recyclages et les synergies au sein d'un territoire (utilisation de déchets environnants et production d'électricité). Pour des exploitations disposant de moins de surface, on pourrait aussi envisager d'utiliser une partie de la chaleur disponible pour sécher les digestats et ainsi produire des engrais organiques qui seraient alors vendus.

\section{5 / Une mobilisation contrastée des principes agro-écologiques}

$\mathrm{Au}$ travers de ces quatre exemples, on constate que les principes agro-écologiques sont mobilisés de façon différente selon 
les SE et selon leur rattachement à une démarche d'AE ou d'EI (tableau 1). Comme on pouvait s'y attendre, la valorisation de la diversité apparaît de manière plus forte dans les systèmes relevant de l'AE, alors que la réduction des pollutions domine en EI. Dans tous les cas, l'économie des intrants et la réduction des pollutions sont deux objectifs qui ressortent systématiquement. Ceci s'explique par les contraintes qui s'imposent aux productions animales : le coût des intrants incite les éleveurs à utiliser de façon parcimonieuse toutes les ressources disponibles et les normes réglementaires les obligent à diminuer les rejets polluants. Les efforts en recherche et développement ont porté aussi très largement sur ces questions et se traduisent in fine par des recommandations pour la mise en place de « bonnes pratiques ». La gestion intégrée de la santé animale est un principe plus rarement mobilisé. Trois hypothèses peuvent être avancées pour expliquer cette situation : $i$ ) le cadre réglementaire (hormis les productions biologiques) autorise le recours à des substances chimiques (traitements vétérinaires), ce qui n'incite pas à rechercher des voies alternatives ; ii) les risques sanitaires sont relativement faibles dans plusieurs SE (particulièrement les systèmes basés sur le pâturage) ; iii) les alternatives aux traitements médicamenteux n'ont été que récemment étudiées, avec des résultats pas toujours mis en œuvre sur le terrain. Toutefois le projet gouvernemental Ecoantibio (http://agriculture.gouv.fr/plan-ecoantibio-2017) devrait changer cette situation. Enfin, le principe visant à préserver la biodiversité ne ressort que dans les SE basés sur le pâturage. Au-delà de maintenir la biodiversité, ces systèmes cherchent aussi à la valoriser en créant les conditions nécessaires à sa pleine expression. Les capacités d'adaptation des animaux et la diversité des ressources sont alors exploitées de façon à accroître la résilience des systèmes (Altieri et Nicholls 2005, Altieri et al 2012).

\section{3 / Implications pratiques pour les systèmes d'élevage : opportunités et freins actuels}

Les systèmes d'élevage affichent une grande diversité en termes de types de production, de niveaux d'intensification et de conditions environnementales. Si des innovations qui se fondent sur les principes agro-écologiques offrent des opportunités pour promouvoir des systèmes productifs en valorisant les processus naturels, elles se heurtent aussi à certains freins. Aussi quelles peuvent être les implications pratiques de la mobilisation des principes agro-écologiques pour les productions animales?
Dans la mesure où cette question dépasse le cadre technique et scientifique des sciences animales, nous apporterons différents éléments de réponse mais poserons aussi des perspectives, avec de nouvelles problématiques et questions à résoudre.

\section{1 / Complémentarités entre agro-écologie et écologie indus- trielle}

Pour produire mieux avec moins d'intrants, nous défendons l'idée de coupler $\mathrm{AE}$ et $\mathrm{EI}$ en raison de leurs contributions complémentaires à la production de services écosystémiques et de produits animaux. Leur socle commun est la mobilisation des concepts de l'écologie, avec l'objectif partagé de minimiser l'empreinte environnementale des SE et de préserver les ressources naturelles. Coupler ces deux approches remet en cause l'opposition classiquement faite entre les deux modèles d'occupation des terres appelés « land sharing » et « land sparing » (Teillard 2012; voir aussi Tscharntke et al 2012 pour l'ensemble du secteur agricole). En élevage, l'AE qui cherche entre autres à valoriser la diversité existant dans les agro-écosystèmes et les ressources non directement valorisables par l'homme pour produire de la viande et du lait s'inscrit dans le modèle du « land sharing ». Elle concourt à préserver des espaces à fort niveau de biodiversité, en maximisant les complémentarités entre ateliers de production et en tirant pleinement parti des services écosystémiques. L'EI relève en revanche d'un modèle d'usage des terres de type « land sparing ». Elle doit permettre d'accroître les niveaux de production, tout en limitant l'usage des ressources naturelles rares, la consommation d'énergie et l'occupation des terres.

\section{2 / Une redistribution des con- traintes par rapport aux systèmes conventionnels}

Dans la plupart des exemples considérés, les deux enjeux majeurs de l'application des principes agro-écologiques à l'échelle du système d'élevage sont : i) de sécuriser les revenus de l'exploitation et ii) de limiter les impacts négatifs de l'élevage sur l'environnement. Il s'agit d'ajuster, à l'échelle du système d'élevage et en considérant des pas de temps longs (plusieurs années et non pas une seule campagne), le rapport entre productivité et intrants. Pour des niveaux de production zootechnique comparables, la forte diminution des intrants permise par la recherche d'une autonomie alimentaire et/ou énergétique élevée aboutit à une augmentation de la valeur ajoutée créée sur l'exploitation (le système ovins présenté dans cet article, Le Rohellec et al 2009, Delaby et al 2014 ce numéro pour les vaches laitières). La diversification des productions stabilise les revenus face aux aléas des marchés, et le bouclage des cycles réduit les pollutions, l'usage d'énergies non renouvelables (exemples IAA, porcs et oies) et le recours à des intrants pour la fertilisation. Cependant, si un des atouts des systèmes relevant de l'AE ou de l'EI est leur autonomie, ils dépendent a priori plus étroitement des conditions pédoclimatiques que les systèmes conventionnels, ce qui augmente les risques pour les éleveurs. Pour sécuriser leur résultat économique, les SE peuvent recourir à des races ou souches locales qui permettent la production de produits sous signe de qualité dans des environnements difficiles (Casabianca et Matassino 2006). De même, travailler sur des objectifs de production zootechnique à moyen terme (sur plusieurs années), en prévoyant de la souplesse dans la conduite des troupeaux et/ou des parcelles, permet de gérer et non plus de subir les aléas auxquels sont soumis ces SE.

Le bilan économique des systèmes relevant de l'AE ou de l'EI est modulé par la possibilité d'obtenir des subventions et par les besoins en main-d'œuvre et en matériel. Dans le cadre des politiques agricoles de l'Union Européenne, l'application des principes agro-écologiques s'accompagne généralement de meilleures performances environnementales qui pourraient donner lieu à des paiements pour services écosystémiques pour les systèmes extensifs ou à une diminution de taxes à la pollution (déjà en place dans certains pays d'Europe du Nord) pour les systèmes intensifs. Le bilan économique peut aussi être limité par la productivité du travail, qui peut imposer une réduction des effectifs d'élevage ou une augmentation de la masse salariale de l'exploitation. L'automatisation des élevages, grâce à de nouvelles technologies et à l'électronique embarquée, peut accompagner (mais pas remplacer) le travail de l'éleveur (Meuret et al 2013). En écologie industrielle, la reproduction en milieu confiné et contrôlé de processus naturels s'appuie sur des technologies avancées nécessitant un matériel spécifique. Le coût généralement élevé de l'investissement initial limite le développement de telles unités.

\section{3 / Une nécessaire réorganisation du travail}

La composante du " travail » est un élément clé des SE mobilisant les principes de l'agro-écologie car même si la quantité de travail n'est pas forcément accrue, sa nature change (Tripp 2008). L'adoption de pratiques agro-écologiques implique une réorganisation du travail avec du temps supplémentaire consacré 
à la surveillance du système, à l'acquisition de nouvelles compétences, en particulier durant la phase de transition, et à l'adaptation du système et de ses pratiques de gestion (intégration de nouvelles productions...). Dès lors, il ne s'agit plus d'appliquer des solutions génériques, mais d'ajuster en continu les décisions à prendre, qui se fondent sur des savoirs et des savoir-faire nés de l'observation minutieuse du fonctionnement du système (Altieri et al 2012, González-García et al 2012). Deux approches pourraient être envisagées pour améliorer la productivité du travail : i) le recours à de nouvelles technologies pour faciliter la collecte, le traitement des informations du système, ou les manipulations de troupeaux (tri, changement de parc...), ii) le développement d'outils (indicateurs d'état, supports pédagogiques et interactifs d'informations, Duru et al 2012) permettant d'accompagner les éleveurs dans l'acquisition de connaissances nouvelles et de compétences spécifiques à son SE.

\section{Conclusion}

Si conceptuellement coupler AE et EI peut permettre de répondre aux défis sociétaux et environnementaux, la transition vers ces nouveaux $\mathrm{SE}$ est à réfléchir selon les contextes environnementaux et les filières. La mobilisation des principes agro-écologiques invite à imaginer des SE en rupture à la fois dans leur organisation et leur gestion, mais aussi dans leurs liens avec l'environnement et la société. L'adhésion et la diffusion des approches agro-écologiques pour les productions animales passent par la mobilisation de réseaux d'éleveurs innovants, la prise en compte des contextes locaux, le développement de soutiens institutionnels et l'identification de marchés permettant une meilleure valorisation de ces produits. La transition vers des systèmes innovants sera favorisée par le développement d'approches participatives dans lesquelles le rôle des acteurs évolue et par la mobilisation d'outils à visée opérationnelle.

Dans l'objectif de concevoir des SE résilients et produisant différents services écosystémiques, le développement de plateformes expérimentales interdisciplinaires est une voie à privilégier, car elle favorise un apprentissage social collaboratif (Biggs et al 2012). De tels dispositifs devraient permettre de capitaliser les savoirs et les savoir-faire pour appréhender de façon holistique les SE de demain à différentes échelles temporelles et spatiales.

\section{Références}

Alikwe P.C.N., Faremi A.Y., Fajemisin A.N., Akinsoyinu A.O., 2011. Performances and nitrogen utilization of West African dwarf goats fed soybean and dried poultry wastebased concentrates as supplements to Cynodon nlemfuensis basal diet. J. Appl. Sci. Environ. Sanit., 6, 181-189.

Altieri M.A., 1987. Agroecology: the scientific basis of alternative agriculture. Boulder: Westview Press. 227p.

Altieri M.A., 2002. Agroecological principles and strategies for sustainable agriculture. In: Agroecological Innovations: Increasing food production with participatory development. NT Uphoff (Ed), Earthscan publication ltd, London, U.K., 40-46.

Altieri M.A., Nicholls C.I., 2005. Agroecology and the search for a truly sustainable agriculture. Basic Textbooks for Environmental Training, United Nations Environment Programme, $291 \mathrm{p}$.

Altieri M.A., Funes-Monzote F.R., Petersen P., 2012. Agroecologically efficient agricultural systems for smallholder farmers: contribution to food sovereignty. Agron. Sustain. Dev., 32, 1-13.

Arroyo J., Fortun-Lamothe L., Dubois J.P., Lavigne F., Auvergne A., 2012. Conduite et gestion des transitions alimentaires chez les oies destinées à la production de foie gras. INRA Prod. Anim., 25, 419-430.

Arroyo J., Fortun-Lamothe L., Auvergne A., Dubois J.P., Lavigne F., Bijja M., Aubin J., 2013. Environmental influence of maize substitution by sorghum and diet presentation on goose foie gras production. J. Clean. Prod., 59, 51-62.

Biggs R., Schlüter M., Biggs D., Bohensky E.L., BurnSilver S., Cundill G., Dakos V., Daw T.M., Evans L.S., Kotschy K., Leitch A.M., Meek C., Quinlan A., Raudsepp-Hearne C., Robards M.D., Schoon M.L., Schultz L., West P.C., 2012. Toward principles for enhancing the resilience of ecosystem services. Ann. Rev. Environ. Res., 37, 421-448.
Bijja M., Dubois J.P., Lavigne F., Auvergne A., Arroyo A., Fernandez X., 2010. Qualité des parcours de palmipèdes : comparaison de la conduite en parcours tournants et en parcours fixe. $9^{\text {imes }}$ Journ. Rech. Palmipèdes à Foie Gras, Bordeaux, France, 197-201.

Bonaudo T., Bendahan A.B., Sabatier R., Ryschawy J., Bellon S., Leger F., Magda D., Tichit M., 2014. Agroecological principles for the redesign of integrated crop-livestock systems. Europ. J. Agronomy, http://dx.doi.org/10.1016/ j.eja.2013.09.010

Bosma R.H., Verdegem M.C.J., 2011 Sustainable aquaculture in ponds: principles, practices and limits. Livest. Sci., 139, 58-68.

Casabianca F., Matassino D., 2006. Local resources and typical animal products. In: Livestock Farming systems - Product quality based on local resources leading to improved sustainability. Rubino R., Sepe L., Dimitriadou A., Gibon A. (Eds). EAAP publication ${ }^{\circ} 118$, Wageningen Academic Publishers, 9-26.

Delaby L., Fiorelli J.L., 2014. Elevages laitiers à bas intrants : entre traditions et innovations. In: Numéro spécial, Quelles innovations pour quels systèmes d'élevage? Ingrand S., Baumont R. (Eds). INRA Prod. Anim., 27, 123-134.

Dourmad J.Y., Rigolot C., Jondreville C., 2009. Influence de la nutrition sur l'excrétion d'azote, de phosphore, de cuivre et de zinc des porcs, et sur les émissions d'ammoniac, de gaz à effet de serre et d'odeurs. INRA Prod. Anim., 22, 41-48.

Dubois J.P., Bijja M., Auvergne A., Lavigne F., Fernandez X., Babilé R., 2008a. Qualité des parcours de palmipèdes : choix des espèces végétales, rendement et résistance au piétinement. $8^{\text {èes }}$ Journ. Rech. Palmipèdes à Foie Gras, Arcachon, France, 107-110.

Dubois J.P., Bijja M., Auvergne A., Lavigne F., Fernandez X., Babilé R., 2008b. Agroforesterie : comportement des oies sous un couvert de noyers et effets sur les performances du verger. $8^{\text {emes }}$ Journ. Rech. Palmipèdes à Foie Gras, Arcachon, France, 111-115.
Dumont B., Fortun-Lamothe L., Jouven M., Thomas M., Tichit M., 2013. Prospects from agroecology and industrial ecology for animal production in the $21^{\text {th }}$ century. Animal, 7, 10281043.

Duru M., Felten B., Theau J.P., Martin G., 2012. A modeling and participatory approach for enhancing learning about adaptation of grassland-based livestock systems to climate change. Reg. Environ. Change, 12, 739-750.

Food and Agriculture Organization (FAO) 2011. Global food lossed and food waste: Extent, causes and prevention. Gustavsson J., Cederberg C., Sonesson U., van Otterdijk R., Meybeck A. (Eds). (Available at http://www. fao.org/docrep/014/mb060e/mb060e00.pdf).

Farruggia A., Dumont B., Scohier A., Leroy T., Pradel P., Garel J.P., 2012. An alternative rotational stocking management designed to favour butterflies in permanent grasslands. Grass Forage Sci., 67, 136-149.

Faruk M.U., Bouvarel I., Meme N., Rideau N., Roffidal L., Tukur H.M., Bastianelli D., Nys Y., Lescoat P., 2010. Sequential feeding using whole wheat and a separate protein-mineral concentration improved feed efficiency in laying hens. Poult. Sci., 89, 785-796.

Frankow-Lindberg B.E., Brophy C., Collins R.P., Connolly J., 2009. Biodiversity effects on yield and unsown species invasion in a temperate forage ecosystem. Ann. Bot., 103, 913-921.

Franzén M., Nilsson S.G., 2008. How can we preserve and restore species richness of pollinating insects on agricultural land? Ecography, 31, 698-708.

Frosch R.A., Gallopoulos N.E., 1989. Strategies for manufacturing. Scientific American, 261, 144-152.

Frosch R.A., 1992. Industrial Ecology: A philosophical introduction. Proc. Nat. Academy Sci. of the USA 89, 800-803.

Gliessman S.R., 1997. Agroecology: Ecological Processes in Sustainable Agriculture. CRC Press. 
Gliessman S.R., 2006. Animals in Agroecosystems. In: Agroecology: The Ecology of Sustainable Food systems. Second Edition. CRC Press, 269-285.

González-García E., Gourdine J.L., Alexandre G., Archimède H., Vaarst M., 2012. The complex nature of mixed farming systems requires multidimensional actions supported by integrative research and development efforts. Animal, 6, 763-777.

Haeckel E.H., 1866. Generelle Morphologie der Organismen. Allgemeine Grunzüge der Organischen Formen-Wissenschaft, mechanisch begründet durch die von Charles Darwin reformirte Descendenz Theorie, 2, Reimer Berlin.

Holm Nielsen J.B., 2010. Bioenergy has a key role to play! Bioenergy in a European context: Potentials, Politics, Developments and Barriers. $6^{\text {th }}$ Int. Bioenergy Conf., Scientific Eng. Centre "Biomass", 1-36.

Hoste H., Jackson F., Athanasiadou S., Thamsborg SM, Hoskin S.O., 2006. The effects of tannin-rich plants on parasitic nematodes in ruminants. Trends Parasitol., 22, 253-261.

IDELE 2010. Consommation d'énergie en élevages herbivores et leviers d'action. Institut de l'élevage, Collection Méthodes et Outils, 92p.

Jouven M., Foulquié D., Benoit M., 2010. Graze rangelands to improve farm performance. Example for an extensive meat sheep farming system. $7^{\text {th }}$ Int. Sem. FAO-CIHEAM Network on Sheep and Goats, 10-12 November 2010, Saragoza, Spain.

Karim M., Little D.C., Shamshul Kabir M.D., Verdegem M.J.C., Telfer T., Wahab M.D.A., 2011. Enhancing benefits from polycultures including Tilapia (Oreochromis niloticus) within integrated pond-dike systems: A participatory trial with households of varying socio-economic level in rural and peri-urban areas of Bangladesh. Aquaculture, 314, 225-235.

Kumaresan A., Pathak K.A., Bujarbaruah K.M., Vinod K., 2009. Analysis of integrated animal-fish production system under subtropical hill agro ecosystem in India: Growth performance of animals, total biomass production and monetary benefit. Trop. Anim. Health Prod., 41, 385-391.

Labbé L., Lefèvre F., Bugeon J., Fostier A., Jamin M., Gaumé M., 2014. Conception d'un système innovant de production de truites en eau recirculée. In : Numéro spécial, Quelles innovations pour quels systèmes d'élevage. INRA Prod. Anim., 27, 135-146.

Lecerf R., 2008. Suivi des changements d'occupation et d'utilisation des sols d'origine anthropique et climatique à l'échelle régionale par télédétection moyenne résolution (Application à la Bretagne). Thèse de doctorat de l'Université de Rennes, France, 2, 325p.

Leprettre S., Auvergne A., Dubois J.P., Babilé R., Lavigne F., 2000. Système d'élevage des oies et performances de croissance. $4^{\text {emes }}$ Journ. Rech. Palmipèdes à Foie Gras, Arcachon, France, 105-108.

Le Rohellec C., Falaise D., Mouchet C., Boutin M., Thiebot J., 2009. Analyse de l'efficacité environnementale et énergétique de la mesure agri-environnementale «Système fourrager économe en intrants » (SFEI), à partir de l'analyse de pratiques de quarante-quatre signataires. Campagne culturale 2006/2007. Renc. Rech. Rum., 16, 109-112.

Lüscher A., Finn J.A., Connolly J., Sebastia M.T., Collins R.P., Fothergill M., Porqueddu
C., Brophy C., Huguenin-Elie O., Kirwan L., Nyfeler D., Helgadóttir A., 2008. Benefits of sward diversity for agricultural grasslands. Biodiversity, 9, 29-32.

Mandonnet N., Tillard E., Faye B., Collin A., Gourdine J.L., Naves M., Bastianelli D., Tixier-Boichard M., Renaudeau D., 2011. Adaptation des animaux d'élevage aux multiples contraintes de régions chaudes. In : Numéro spécial, Elevage en régions chaudes. Coulon J.B., Lecomte P., Boval M., Perez J.M. (Eds). INRA Prod. Anim., 24, 41-64.

Martin C., Morgavi D.P., Doreau M., 2010. Methane mitigation in ruminants: from microbe to the farm scale. Animal, 4, 351-365.

Merrifield D.L., Dimitroglou A., Foey A., Davies S.J., Baker R.T.M., Bøgwald J., Castex M., Ringø E., 2010. The current status and future focus of probiotic and prebiotic applications for salmonids. Aquaculture, 302, 1-18.

Meuret M., Tichit M., Hostiou N., 2013. Elevage et pâturage « de précision » : l'animal sous surveillance électronique. Le courrier de l'Environnement de l'INRA, 63, 13-24.

Molénat G., Foulquié D., Autran P., Bouix J., Hubert D., Jacquin M., Bocquier F., Bibe B., 2005. Pour un élevage ovin allaitant performant et durable sur parcours : un système expérimental sur le Causse du Larzac. INRA Prod. Anim., 18, 323-338.

Mounier L., Colson S., Roux M., Dubroeucq H., Boissy A., Ingrand S., Veissier I., 2006. Links between specialization in the finishing of bulls, mixing, farmers' attitudes towards animals and the production of finishing bulls: a survey on French farms. Anim. Sci., 82, 561-568.

Neori A., Chopin T., Troell M., Buschmann A.H., Kraemer G.P., Halling C., Shpigel M., Yarish C., 2004. Integrated aquaculture: rationale, evolution and state of the art emphasizing seaweed biofiltration in modern mariculture. Aquaculture, 231, 361-391.

Nhan D.K., Milstein A., Verdegem M.C.J., Verreth J.A.V., 2006. Food inputs, water quality and nutrient accumulation in integrated pond systems: A multivariate approach. Aquaculture, 261, 160-173.

Nhan D.K., Phong L.T., Verdegem M.J.C., Duong L.T., Bosma R.H., Little D.C., 2007. Integrated freshwater aquaculture, crop and livestock production in the Mekong delta, Vietnam: determinants and the role of the pond. Agric. Syst., 94, 445-458.

Petersen S.O., Sommer S.G., Béline F., Burton C., Dach J., Dourmad J.Y., Leip A., Misselbrook T., Nicholson F., Poulsen H.D., Provolo G., Sorensen P., Vinnerås B., Weiske A., Bernal M.P., Böhm R., Juhász C., Mihelic R., 2007. Recycling of livestock manure in a whole-farm perspective. Livest. Sci., 112, 180-191.

Phong L.T., van Dam A.A., Udo H.M.J., van Mensvoort M.E.F., Tri L.Q., Steenstra F.A., van der Zijpp A.J., 2010. An agro-ecological evaluation of aquaculture integration into farming systems of the Mekong Delta. Agric. Ecosyst. Environ., 138, 232-241.

Phong L.T., de Boer I.J.M., Udo H.M.J., 2011. Life cycle assessment of food production in integrated agriculture-aquaculture systems of the Mekong Delta. Livest. Sci., 139, 80-90.

Prein M., 2002. Integration of aquaculture into crop-animal systems in Asia. Agric. Syst., 71, 127-146.

Puillet L., Martin O., Sauvant D., Tichit M., 2010. An individual-based model simulating goat response variability and long term herd performance. Animal, 4, 2084-2098.

Rahman M.M., Verdegem M.C.J., Nagelkerke L.A.J., Wahab M.A., Milstein A., Verreth J.A.J., 2006. Growth, production and food preference of rohu Labeo rohita (H.) in monoculture and in polyculture with common carp Cyprinus carpio (L.) under fed and non-fed ponds. Aquaculture, 257, 359-372.

Rigolot C., Espagnol S., Robin P., Hassouna M., Béline F., Paillat J.M., Dourmad J.Y., 2010. Modelling of manure production by pigs and $\mathrm{NH}_{3}, \mathrm{~N}_{2} \mathrm{O}$ and $\mathrm{CH}_{4}$ emissions. Part II: effect of animal housing, manure storage and treatment practices. Animal, 4, 1413-1424.

Sabatier R., Doyen L., Tichit M., 2010. Modelling trade-offs between livestock grazing and wader conservation in a grassland agroecosystem. Ecol. Model., 221, 1292-1300.

Sabatier R., Doyen L., Tichit M., 2014. Heterogeneity and the trade-off between ecological and productive functions of agro-landscapes: A model of cattle-bird interactions in a grassland agroecosystem. Agric. Syst., 126, 38-49.

Scohier A., Ouin A., Farruggia A., Dumont B., 2013. Is there a benefit of excluding sheep from pastures at flowering peak on flowervisiting insect diversity? J. Insect. Conserv., 17, 287-294

Soder K.J., Sanderson M.A., Stack J.L., Muller L.D., 2006. Intake and performance of lactating cows grazing diverse forage mixtures. J. Dairy Sci., 89, 2158-2167.

Teillard F., 2012. Reconciling food production and biodiversity in farmlands: the role of agricultural intensity and its spatial allocation. Université Paris Descartes, 179p.

Tichit M., Hubert B., Doyen L., Genin D., 2004. A viability model to assess the sustainability of mixed herds under climatic uncertainty. Anim. Res., 53, 405-417.

Tichit M., Puillet L., Sabatier R., Teillard F., 2011. Multicriteria performance and sustainability in livestock farming systems: Functional diversity matters. Livest. Sci., 139, 161-171.

Tripp R., 2008. Agricultural change and lowinput technology. In: Agricultural systems: agroecology and rural innovation for development. Snapp S., Pound B. (Eds). Elsevier Academic Press Publication, Amsterdam, The Netherlands, 129-160.

Tscharntke T., Clough Y., Wanger T.C., Jackson L., Motzke I., Perfecto I., Vandermeer J., Whitbread A., 2012. Global food security, biodiversity conservation and the future of agriculture intensification. Biol. Conserv., 151, 53-59.

Veen P., Jefferson R., de Smidt J., van der Straaten J., 2009. Grasslands in Europe of high nature value. KNNV Uitgeverij, NHBS Distribution, Totnes, Devon, UK.

Wezel A., Soldat V., 2009. A quantitative and qualitative historical analysis of the scientific discipline agroecology. Int. J. Agric. Sustainability, 7, 3-18.

World Health Organization (WHO), 2006. Guidelines for the safe use of wastewater, excreta and greywater. Volume 3. Wastewater and excreta use in aquaculture. Switzerland: Geneva, 1-114. 


\title{
Résumé
}

L'agro-écologie et l'écologie industrielle, qui mobilisent les concepts de l'écologie, sont deux approches complémentaires pour répondre aux enjeux économiques et environnementaux auxquels sont confrontés les systèmes d'élevage. L'agro-écologie cherche à amplifier les processus naturels pour promouvoir des systèmes productifs moins dépendants des intrants, valorisant la diversité animale et végétale et produisant différents services écosystémiques. L'écologie industrielle explore le bouclage des cycles (matière, énergie) pour économiser les ressources nécessaires à la production, et diminuer les pollutions dans des systèmes très contrôlés et ayant un faible lien au sol. Jusqu'ici, les systèmes d'élevage ont été largement écartés des démarches agro-écologiques. Nous proposons et analysons cinq principes agro-écologiques applicables à l'élevage $: i$ ) développer des pratiques de gestion intégrée pour améliorer la santé animale, ii) utiliser des ressources naturelles et des coproduits pour diminuer les intrants nécessaires à la production, iii) optimiser le fonctionnement des systèmes d'élevage pour réduire les pollutions, $i v)$ valoriser la diversité dans les élevages pour renforcer leur résilience, et v) adapter les pratiques d'élevage pour préserver la biodiversité dans les agro-écosystèmes et les services écosystémiques associés. A partir de quatre études de cas, nous décrivons comment ces principes se combinent et permettent d'atteindre de meilleures performances économiques et environnementales. Les opportunités et freins actuels pour le développement de systèmes relevant de l'agroécologie ou de l'écologie industrielle sont ensuite discutés en évoquant la motivation des éleveurs à développer des systèmes autonomes et la réorganisation du travail nécessaire pour que s'opèrent de tels changements.

\begin{abstract}
Agroecology and industrial ecology: two complementary alternatives for animal production in the $21^{\text {st }}$ century

Agroecology and industrial ecology can be viewed as complementary means for the design of sustainable animal farming systems: agroecology by stimulating natural processes to reduce inputs, and using diversity to increase system resilience and provide ecosystem services. Industrial ecology mainly aims to close system loops within a territory, thereby reducing demand for raw materials and lowering pollution. Animal farming systems have so far been ignored in most agroecological thinking. We propose five principles applicable to animal production systems: $i$ ) adopting health management practices that minimize the use of chemical drugs, ii) decreasing the inputs needed for production by using grazing lands and by-products, iii) decreasing pollution by optimizing the functioning of farming systems, iv) enhancing diversity within animal production systems to strengthen their resilience, and v) preserving biological diversity and associated ecosystem services by adapting management practices. Based on four case studies, we show how these different principles can be combined to improve economic and environmental performance. We discuss opportunities and resistance to change what needs to be considered for the scaling-up of agroecological systems. Among these, we stress the motivation of farmers to break input dependency and the question of workload.
\end{abstract}

THOMAS M., FORTUN-LAMOTHE L., JOUVEN M., TICHIT M., GONZÁLEZ-GARCÍA E., DOURMAD J.-Y., DUMONT B., 2014. Agro-écologie et écologie industrielle : deux alternatives complémentaires pour les systèmes d'élevages de demain. In: Numéro spécial, Quelles innovations pour quels systèmes d'élevage? Ingrand S., Baumont R. (Eds). INRA Prod. Anim., 27, 89-100. 